\title{
América Latina: Una historia de violencia heredada. Revisión bibliográfica.
}

\section{Latin America: history of inherited violence. Review paper.}

\author{
Blanca Andrea Ortega Marín ${ }^{1}$ https://orcid.org/0000-0002-6821-8239
}

*Correspondencia a: blancaortega@utectulancingo.edu.mx

\author{
${ }^{1}$ Área de \\ Electromecánica \\ Industrial Universidad \\ Tecnológica de \\ Tulancingo, Hidalgo, \\ México \\ Recibido: Oct. 2020, \\ Aceptado: Nov. 2020 \\ Aspectos éticos: \\ La autora declara que \\ no existe conflicto de \\ interés \\ en la publicación de \\ este artículo.
}

\section{RESUMEN}

Introducción: América Latina a cinco décadas de seguir las políticas económicas y fiscales del Fondo Monetario Internacional y del Banco Mundial, que la llevarían al crecimiento y desarrollo y en las que los Estados-gobiernos se centraron para construir la democracia, sin tener el efecto deseado, al contrario, la región está viviendo en la violencia más profunda y creciente, sumado al reto de la COVID-19. Objetivo: Conocer las causas que originaron esta situación, que afecta a la población vulnerable, que sufre la violencia en varios entornos y con efectos diversos. Metodología: Cualitativa (1970-2020); en lo histórico, económico, social, de desarrollo, de crecimiento y bienestar en la región, siguiendo los postulados de Amartya Sen del año 2000 con relación al ejercicio de las libertades de la población y su bienestar. Se prefirieron las revisiones, estados del arte, estudios y análisis. Resultados: Se encontró que el modelo económico colapsó desde 1980 pues fundamentó su base económica solo en los recursos naturales. Continuó la dependencia externa. Las políticas económicas, fiscales y asistenciales tampoco fueron efectivas. La población se quedó sin educación y empleo, generando migración creciente, pobreza extrema y vulnerabilidad. Centroamérica es la zona más golpeada ya que sus habitantes son mayoritariamente rurales e indígenas. Actualmente se sigue el mismo modelo. Regresó la violencia de las fuerzas policiales y esta se detonó también en la población como mecanismo de defensa y sobrevivencia. Conclusiones: Los hallazgos demuestran el fracaso del modelo; las políticas nunca consideraron la capacidad contributiva real, ni las demandas de educación, empleo, seguridad y bienestar. Finalmente, los efectos de la pandemia en la región, corroboran lo endeble del sistema de salud, la fragilidad de las economías y a nivel social, el inminente incremento de población vulnerable con futuro incierto.

\section{PALABRAS CLAVE}

Latinoamérica y COVID-19, Pobreza y violencia, Vulnerabilidad y violencia, Latinoamérica y violencia, Pobreza y desigualdad, Revisión Bibliográfica.

\section{CITAR COMO}

Ortega-Marín BA. América Latina: Una historia de violencia heredada. Revisión Bibliográfica. Rev. cienc. forenses Honduras. 2020; 6(2): 10-23 DOI: https://doi.org /10.5377/rcfh.v6i2.10707 


\section{ABSTRACT}

Introduction: Latin American, in five decades following the economic and fiscal politics from the FMI and BM, that would reach them to grow and develop and the Government-States focused on building the democracy, the region is living in the deepest and growing violence and, the COVID-19 challenge, in an emergency status. Objective: This generated the interest in knowing the causes that originated the situation that affects the vulnerable population that suffers from violence in various sceneries with diverse effects. Methodology: was qualitative (1970-2020); related to historic, economic, social, development, growth and wellbeing aspects in the region, following Amartya Sen's postulates (2000) in reference to the population liberties and their well-being. The revisions, art state, studies and analysis were selected. Results: The economic model was collapsed since 1980 because its economic basys was the natural resources. The external dependency continued. The economic, fiscal and assistential politics were ineffective. The population remained without education and job, generating growing migration, extreme poverty and vulnerability. Central America has been the most affected area due to its inhabitants are indigenous. At present the same model continues. The police force violence came back and it also rose in the population as defense and survival. Conclusion: The facts show the model crash, the politics never considered the real contributive capacity, neither the education demands, job, security and well-being. Finally, the COVID-19 effects in the region, prove the weak health care system, the fragility of the economies and socially, the imminent increasing of vulnerable population, with an uncertain future.

\section{KEYWORDS}

\footnotetext{
1 Se incluyó el término Estados-gobiernos para referirse al conjunto de órdenes y/o instancias de gobierno de los países de América Latina.
}

Latin America and COVID-19, Poverty and violence, Vulnerability and violence, Latin America and violence, Poverty and inequality, Bibliographic review.

\section{INTRODUCCIÓN}

América Latina ( $A L)$ o Latinoamérica (LA) está integrada por 20 países, de gran riqueza natural, diversidad biológica y que aportan arriba del 40 por ciento de la biodiversidad al planeta tierra ${ }^{1}$. Su población actual es de 644,137,670 millones de personas de las que 42.5 millones se encuentran en situación de hambre según las Naciones Unidas para la Alimentación y la Agricultura (FAO), y viven con problemas de salud y desnutrición debido a sus carencias de alimentación y además se posicionó en el primer sitio en materia de violencia mundial 2,3,4. Esta investigación es una revisión históricoeconómico-social que tiene la finalidad de realizar una aproximación acerca de qué sucedió en Latinoamérica para que, a pesar de su riqueza natural, ocupe el primer sitio en violencia y pobreza en el mundo ${ }^{5}$, y como las acciones de los Estadosgobiernos $^{1 *}$ y de los organismos internacionales en la región entre 1970-2020, orientadas a su avance, con ofertas laborales y encaminando hacia una vida con los satisfactores cubiertos $^{6}$, a cinco décadas de operarlas, los resultados están lejos de alcanzarse.

\section{METOdOLOGÍA DE BÚSQUEDA}

La revisión es de carácter cualitativo y para llevarla a cabo se utilizó una metodología que incluyó la selección de documentos históricos, económicos (considerando el desarrollo y crecimiento), políticos, sociales (incluyendo el bienestar) en LA, cuyos postulados se sustentaron en información de las áreas mencionadas, en el análisis de varias fuentes $y / 0$ el resultado de hechos sociales 
comprobados y/o apoyados con datos estadísticos. Para garantizar lo anterior, se definieron criterios para la búsqueda de información:

1. Se seleccionaron publicaciones con análisis de las áreas ya indicadas, y/o que contuvieran alguna o varias de ellas, con la finalidad de disponer de la mayor información posible.

2. Se priorizó el acopio de materiales catalogados como análisis, estudios, estados del arte y revisión; a fin de contar con un mayor número de opiniones de estudiosos en el tema y que dieran mayor solidez a la discusión y conclusiones de esta investigación.

3. Se eligieron documentos con una antigüedad máxima de cinco años (hacia atrás), a excepción de aquellos considerados históricos que daban cuenta de sucesos relacionados con las dimensiones de estudio a lo largo del tiempo, aunque se hubieran publicado con más años de antigüedad.

4. Se efectuó la búsqueda en portales académicos en español y de libre acceso; por tratarse de una investigación sobre LA.

5. Se seleccionaron publicaciones académicas y/o de universidades, y se consideraron las de organismos internacionales que aportaron información histórica; y/o explicativa; sustentada en datos estadísticos $y$, a su vez, incluyera la participación de otros estudiosos y/o académicos para su realización.

El trabajo se organizó en varias etapas:

I. Se definieron las palabras clave que tuvieran correspondencia con la finalidad de la revisión: análisis, estudios, estados del arte y/o revisión.

II. Se acopiaron los materiales disponibles relacionados con los temas históricos, económico, político, social, bienestar, crecimiento y desarrollo político, social, bienestar, crecimiento y desarrollo ya mencionados.
III. Se seleccionaron las publicaciones, según el nivel de aporte de los documentos, siguiendo los criterios definidos.

IV. Se integraron archivos con las publicaciones elegidas, por área, para su revisión.

V. Se extrajeron los apartados coincidentes con los criterios definidos, para reconstruir los hallazgos de acuerdo con los temas de interés.

\section{Sobre la búsqueda}

La información disponible conforme a las dimensiones y criterios previstos arrojó arriba de 80 publicaciones y algunas se desecharon por resultar de algún país en particular. Se localizaron tres revisiones entre: 1980-2000, 1980-2016 y 19952004. Dos de ellas contuvieron cinco décadas de información, pero solo en una o dos de las áreas de interés, y la tercera abarcó solo una década. El resto de publicaciones fueron de los años 2007, 2008, 2010, 2015, 2016, 2017, 2018, 2019 y 2020. Cabe mencionar que muchas de ellas -sin incluirlo en el título-, al momento de leerlas se basaron en numerosos documentos $y$, en particular, se encontró una cantidad mayor sobre la violencia. Con base en el contenido aportado se fueron reconstruyendo los hallazgos; según lo estipulado.

\section{Antecedentes}

La colonización del territorio e islas que conforman AL inició en el siglo XV y la colonia se reconoció como tal a partir del siglo XVI por los españoles que, embelesados por las particularidades del entorno natural, decidieron establecerse en la zona mediante la sumisión forzada, esclavizando a los nativos, las guerras fabricadas y/o la liquidación para apoderarse de las tierras (que orientarían al área agropecuaria), la riqueza mineral (oro y plata) y la fuerza laboral nativa que fue lanzada de su territorio ${ }^{7}$. En la revisión de las obras de Fray Bartolomé de las Casas destacan las innumerables 
denuncias por los eventos comentados, que alentaron la redacción de los Tratados de Burgos y La Nueva Ley de Indias, que instaba a encomenderos y obispos a desistir de las acciones señaladas, sin conseguirlo. En especial en el libro "La Apologética historia sumaria", de las Casas, enfatiza en la geografía de la región, las peculiaridades de las personas, su organización, y forma de vida en las llamadas Indias Occidentales ${ }^{7}$, destacando que tenían una forma diferente de ver la vida y su hábitat. Los siglos de "conquista" fecundaron una gran contradicción: aunque Latinoamérica prodigó la riqueza que hizo posible el desarrollo del capitalismo mundial ... su cosecha... fue quedarse en el atraso, en términos del avance previsto y generar miseria en los pobladores a lo largo del tiempo ${ }^{8}$.

\section{La evolución histórica del concepto de desarrollo}

Hablar del término desarrollo implica partir del reconocimiento -en 1940- de las necesidades de insumos de los países europeos para sus actividades productivas (Primera Declaración Interaliada ${ }^{9}$ y en la Carta del Atlántico) ${ }^{10}$, mismo que es retomado en la Conferencia de San Francisco ${ }^{11}$, cuando dieron vida a la Organización de Naciones Unidas (ONU) ${ }^{12}$. Es en este contexto que los países participantes asignaron a AL el papel de "proveedor" de diversos recursos; tanto a Europa como a EEUU (en diferentes momentos). En la Segunda Guerra Mundial se retoma el término; en la conformación de las teorías del desarrollo, dilucidando los componentes de la economía, los obstáculos de las estructuras económicas de los designados "países subdesarrollados", "dependientes", "periféricos o emergentes", que fueron congregados en el llamado Tercer Mundo ${ }^{13}$. La Conferencia Económica para América Latina (CEPAL), abrigó la discusión en cuanto a industrializar a la región mediante la sustitución de importaciones y fomentar las exportaciones, como su estrategia económica ${ }^{14}$.
Casi al mismo tiempo (mediados del siglo XX) se estipuló que el objetivo del desarrollo económico era el crecimiento y acumulación de capital ${ }^{15}$, se planteó el enfoque Neo-Clásico, donde el desarrollo significó el paso de la sociedad estancada y en subsistencia a una dinámica y capitalista; centrada en el sector emprendedor ${ }^{16}$, conforme lo documenta la Teoría de las Etapas del Crecimiento Económico ${ }^{17}$. Mientras tanto, en AL (años 60-70), diversos pensadores, examinaron la situación socioeconómica de sus países, dando como resultado el Modelo Desarrollista de la CEPAL ${ }^{18-21}$. El Estadogobierno es el propulsor del desarrollo, bajo la perspectiva economicista y latinoamericana, reconociendo que éste está detenido, sin generar empleo ni distribuir el ingreso.

El modelo desarrollista fue cuestionado por la Teoría de la Dependencia (en la década de los 70) construida por algunos latinoamericanos ${ }^{22-26}$ cuyo enfoque se sustentó en el pensamiento marxista. Calificaron al modelo como economicista, destacando que AL fue el abastecedor de materias primas e insumos que hicieron posible la industrialización de los países centrales, creándose clases oligárquicas endógenas; que sometieron las relaciones supeditadas a su conveniencia, revelando la continuidad en la dominación de países desfavorecidos en los que unos cuantos se beneficiaban ${ }^{27}$.

Será la crisis del capitalismo en EEUU (década de los 80) y, previo al surgimiento de la Teoría de la Regulación en Francia ${ }^{28}$ y la Escuela Francesa de la Regulación ${ }^{29}$, que lleva a retomar el enfoque teórico keynesiano y se propició la coyuntura para la aplicación de las políticas neoliberales del Fondo Monetario Internacional (FMI) en AL y se inicia el camino para lograr el desarrollo ${ }^{30}$.

Los Estados-gobiernos en la región establecieron nuevas políticas económicas y laborales que, -desde 
su inicio y hasta ahora- han significado un largo camino en el que continúa pendiente que los latinoamericanos disfruten el progreso. El aumento de la pobreza alentó la migración, especialmente en Centroamérica ${ }^{31}$, convirtiendo a la región en la más desigual, insegura y violenta del mundo ${ }^{32}$.

A partir del mes de febrero de 2020; la pandemia de la COVID-19; ha traído para América Latina retos profundos en materia de salud, con repercusiones económicas inmediatas y a nivel social; desintegrando a las familias, dejando sin empleo a muchas personas, deteniendo las economías para disminuir los contagios y, particularmente, dejando al descubierto la precariedad en la que operan las instituciones de salud. Todo esto expresado en la ausencia de personal especializado, las carencias en equipo, en medicamentos e instalaciones. Esta circunstancia está mostrando el crudo significado de la pobreza en el que se encuentran dos terceras partes de la población latinoamericana, viviendo una situación muy delicada ${ }^{33}$. Asimismo, entre los resultados desfavorables se prevé un número mayor de pobres ${ }^{34}$, las economías en los países se verán frenadas, arriesgando el futuro de los países $^{35}$.

\section{RESULTADOS}

En 1970 los Estados-gobiernos latinoamericanos permanecieron con el mismo modelo de sustitución de importaciones, hasta que surgieron los altibajos económicos mundiales que sumieron a los países en los efectos del mercado, eliminando la posibilidad de continuarlo, y llevándolos a la llamada década perdida de los 80's.

La coyuntura mostró la debilidad económica; resultado de la dependencia externa $y$, aunque representó la oportunidad para construir un camino propio y diferente hacia el desarrollo y crecimiento en cada país de la región, continuaron bajo las pautas del mercado global, y las directrices del FMI y del Banco Mundial (BM).
Las políticas fiscales y la recaudación fueron aceptables en una primera etapa, pero a partir de 1990 tuvieron pocos resultados; derivado de las características desiguales en los ingresos de los contribuyentes y de los propios sectores productivos.

A nivel social, los efectos del bache económico, se manifestaron en la pérdida de las posibilidades laborales para quienes ya desempeñaban alguna actividad, llevando a más población a la pobreza, para los adolescentes a quedarse sin más oportunidades educativas que el nivel secundario, sin opciones laborales al aplicarse las estrategias de selectividad en la población privilegiada, llevando poco a poco a un mayor deterioro en las condiciones de vida en general.

Lo anterior obligó a la población económicamente activa (PEA) desempleada y a los jóvenes sin opciones a dedicarse a las actividades informales generadas por ellos mismos, con salarios muy bajos y sin ningún tipo de incentivo y/o seguridad social; por estar fuera del esquema económico reconocido en cada país. Al mismo tiempo la PEA atestiguó lo que ofrecían las ciudades, pero inhabilitados para acceder a esos satisfactores por falta de recursos y/o mayor preparación académica, viviendo ahora excluidos de las oportunidades que aparentemente eran para todos.

En el 2000 se impulsaron -como resultado de las iniciativas del Programa de Naciones Unidas para el Desarrollo (PNUD)- las acciones Ilamadas Metas del Milenio, que se tradujeron en políticas públicas orientadas al bienestar social y al desarrollo de la población; coordinadas por los Estados-gobiernos de cada país. A dos décadas de operación, sus frutos han sido pocos, poniendo en duda desde sus objetivos, como la metodología empleada por las instituciones para ejecutarlas, la preparación y experiencia de los encargados de operarlos. 
Todo ello propició la desconfianza y temor de la población pobre, -ahora marginada y discriminadahacia los Estados-gobiernos, en particular al reaparecer las muestras de control violento de las fuerzas públicas, desdibujando el camino recorrido para alcanzar la democracia y, casi simultáneamente, surgieron grupos de jóvenes para defenderse de los excesos policíacos. En algunos países se iniciaron las actividades ilícitas, atentando contra la vida de sus congéneres (mediante el secuestro, la extorsión o las amenazas a cambio de prebendas y/o dinero) y/o involucrándose en actividades delincuenciales a nivel internacional que, poco a poco, fueron incrementado la violencia para "ganar un espacio" en los propios lugares donde se asentaron; o frente a grupos rivales. Poco a poco se fueron sumando un mayor número de jóvenes y mujeres a estas actividades.

De esta forma la violencia adquirió un doble papel: como defensa ante los abusos de las autoridades y poco a poco se fue normalizando como otra manera de vivir y como forma de obtener recursos para satisfacer las diversas carencias que esa población había venido acumulando en los últimos cincuenta años (donde están involucradas ya tres generaciones). Por su parte, la población que se quedó al margen, sobrevive en violencia, sufriéndola en lo físico, lo laboral, de género, en lo económico, en lo social y en lo emocional.

\section{DISCUSIÓN}

La década perdida trajo consigo duras lecciones para la región. Al continuar siendo rural la base económica de exportación ${ }^{36}$ sus posibilidades de crecimiento en el corto plazo fueron improbables, dejando al descubierto la debilidad de los Estadosgobiernos y la gran fragilidad laboral-social, cuyos efectos marcaron a Latinoamérica. La coyuntura fue propicia para reconstruir el desarrollo, y/o sentar nuevas bases económicas; a partir del reconocimiento de la gran riqueza natural y proponiendo nuevas posibilidades para combinarlas con actividades productivas de carácter local; que fueran fortaleciendo las capacidades productivas, retomando la experiencia previa. Sin embargo, AL careció de propuestas novedosas encaminadas al surgimiento de nuevos sectores que ampliaran su potencial. Tampoco regresaron a actividades antaño exitosas, ni reactivaron las catalogadas como primordiales para los mercados internos, ni generaron fortalezas endógenas que dieran un respiro al estancamiento. En las ciudades medianas y en el campo, al existir pocas opciones de progreso locales, el hambre fue orillando al abandono de las actividades agropecuarias, estimulando nuevamente la migración ante la profundización de la pobreza en los sectores con menos oportunidades educativas, y lo mismo ha sucedido con los que habitan en ciudades urbanas o metropolitanas, imposibilitados para vivir con un salario de uno o dos dólares al día ${ }^{37}$ sumándose a la diversidad de problemáticas $y$, particularmente, continuar motivando la migración en los países centroamericanos $^{38}$ cuya mayoría de habitantes son de origen rural y/o indígena.

La productividad latinoamericana tampoco se recuperó ${ }^{39}$, ni ha mejorado el salario familiar que se ha mantenido en cuatro dólares diarios por familia ${ }^{32,41}$ producto de la continuidad de las medidas económicas de los organismos financieros internacionales; institucionalizadas y aplicadas por los Estados-gobiernos.

Las políticas fiscales sólo han incorporado entre 25 y 50 por ciento de la $\operatorname{PEA}^{41}$ y son las ciudades más pobladas y/o con recursos minerales y/o extractivos las que han impulsado actividades económicas representativas y han podido apreciar cierto crecimiento, mediante inversiones extranjeras ${ }^{42}$. Sin embargo, al estarinmersas en el mercado global, 
continúan dependiendo del exterior, reproduciendo el mismo esquema de manera cíclica.

A la fecha, la PEA, nunca ha sido reconocida por el papel que representa ${ }^{43}$ ni ha tenido oportunidad de participar en el rediseño de su propia visión de florecimiento y, en particular, para la generación de jóvenes que sólo pudo formarse a nivel básico y un bajo porcentaje a nivel secundaria y preparatoria ${ }^{40}$, que quedó fuera de toda posibilidad ante la tendencia del modelo hacia la tecnología intelectual y la nueva intelligentsia en las universidades ${ }^{44}$, que demandaron la refuncionalización de la mano de obra y la creciente especialización.

Los excluidos y sin opciones han pasado del sufrimiento ante la falta de oportunidades a la pobreza, a la pobreza extrema y ahora a la vulnerabilidad $^{40}$. Como resultado, han vivido en desnutrición, enfermedades ${ }^{45}$, y en especial la difícil situación socioeconómica y política ${ }^{46}$ que ha vivido la población, en particular la centroamericana desde hace varias décadas ${ }^{47}$. Todo ello, propició un distanciamiento y desconfianza creciente de la población hacia los Estados-gobiernos ${ }^{39}$, dudando de su finalidad ${ }^{32}$ ante los nulos beneficios tangibles, llevando a las personas a ser violentos ${ }^{48}$ desde edades tempranas 49,50 ...sumarse a... grupos 0 pandillas ${ }^{51}$ como forma de sobrevivencia 52,5354 , sin haber comprendido las razones por las que nunca tuvieron acceso a "las oportunidades" que vieron a su alrededor a lo largo del tiempo, o formar parte y disfrutar de una familia con una buena calidad de vida, contar con una casa, acceder a la educación, tener una alimentación suficiente, desempeñar un empleo bien pagado, decidir su vida y/o su futuro. Todo ello se corrobora en los tres últimos quinquenios, mediante los índices de desarrollo humano que continuaron a la baja y explican el incremento de la violencia entre 2000 y $2010^{55}$ y al 2019 para AL, en promedio, apenas alcanzan el $0.589^{56}$. Sumando a lo anterior, actualmente la región vive en la tragedia sanitaria del COVID19, encontrándose en el umbral de una vida incierta y desprotegida, en particular los cerca de 140 millones de personas que se encontraban en el sector informal ${ }^{57}$.

\section{CONCLUSIONES}

Los hallazgos de esta revisión en las dimensiones histórico-social-económica, demuestran que la población en AL continúa sin alcanzar el crecimiento, el desarrollo y el bienestar prometidos desde 1970, y al 2020 se corrobora el fracaso del modelo capitalista centrado en el mercado, así como de las políticas fiscales y socio-asistenciales aplicadas en la región.

Los hechos dan cuenta que la memoria que se ha ido construyendo y repitiendo -a través del tiempo- en la población menos favorecida, es el maltrato, la discriminación, la exclusión y la pobreza que se han profundizado con el paso del tiempo; se normalizaron y los han confinado a vivir en situación de vulnerabilidad.

Latinoamérica requerirá su reconstrucción en el corto plazo, debiendo partir del reconocimiento del actual potencial de los recursos de cada país, de su importancia económica ${ }^{58}$ por sus aportes y alcances de carácter nacional, regional y/o mundial y junto con la población, trazar las estrategias más viables para la economía de cada país.

Es la coyuntura para retomar la memoria productiva del territorio y mediante la reconstrucción geográfica, determinar qué proyectos locales es posible impulsar que impacten lo regional $\mathrm{y} / \mathrm{o}$ reactiven actividades viables ${ }^{27}$; recuperando sus vocaciones productivas, con enfoque de gestión sostenible y coadyuvar en la disminución de la grave inseguridad alimentaria que prevalece en Latinoamérica ${ }^{59}$. Los nuevos Estados-gobiernos deben basarse en un sistema íntegro y transversal 
incluyendo a los diferentes órdenes de gobierno, cuya regulación sea consistente con las competencias posibles, que frenen el abuso e ilegalidad en los bienes y servicios que proporcionan $^{32}$.

Los empleos que oferten deben ser de calidad, con sueldos suficientes para sufragar los requerimientos de las familias y contar con prestaciones acordes. Es urgente rediseñar las políticas educativas con base en datos actuales, que amplíen las posibilidades de acceso a la educación en los diferentes niveles y población ${ }^{40}$, a fin de que al término; sea posible emplearse ${ }^{60}$ y se cimente en condiciones laborales aceptables para todos ${ }^{32}$. En lo tributario, las nuevas políticas y los impuestos deben ser equitativos, tomando como base las posibilidades contributivas de la PEA, orientarse a horizontalidad, favoreciendo la aceptación de los contribuyentes ${ }^{61}$. En cuanto a la atención social y a fin de iniciar la recuperación de la confianza de la población, es recomendable que las acciones sean definidas por los beneficiarios, conforme a sus intereses y lo que desean emprender y alcanzar a largo plazo. Que la atención sea integral; incluyendo todo aquello a lo que tienen derecho y que se orienten a minimizar la desigualdad, en especial en los más vulnerables ${ }^{62}$. Se sugiere también que definan con los interesados las acciones que mejoren sus condiciones de vida y bienestar, incluyendo a todos los sectores y se atienda a todos de forma igualitaria ${ }^{63}$, especialmente a los jóvenes y estudiantes ${ }^{39}$.

En cuanto a la violencia, comenzar por erradicar las acciones que la promueven desde los Estadosgobiernos ${ }^{64}$, analizar por país el nivel que ha alcanzado la ausencia del estado de derecho en AL, cuyo índice se localizó por debajo del 0.5 en la mayoría de ellos ${ }^{32}$. Comprender cómo el recurrente entorno de penuria llevó a delinquir ${ }^{65}$, o como camino para mejorar, y lo adoptaron como modus

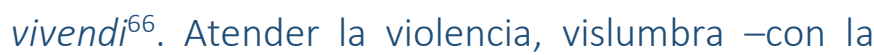

información disponible- tres posibilidades: La primera, previniéndola desde la infancia hasta la adultez, reenfocando su atención, y los efectos psicológicos en quienes la sufren ${ }^{67}$. La segunda mediante la atención a las personas violentas (el contexto organizativo ${ }^{68}$ y el tercero a las personas afectadas (incluyendo lo sexual), lo emocional ${ }^{69}$, lo vivido $50,70,71$, y lo que afecta ${ }^{72}$ ).

Finalmente, ante el futuro incierto que la pandemia representa para América Latina al mismo tiempo es una oportunidad única para reescribirse, desde la perspectiva de la propia población, apoyados de un equipo multidisciplinar, con perspectiva bioética, social, valores éticos y visión de futuro sostenible.

\section{REFERENCIAS BIBLIOGRÁFICAS}

1. Mapa de los 17 países megadiversos en el mundo. INFOBAE. 2020 [Internet]. América. [citado 3 de julio de 2020]. Disponible en: https://www.infobae.com/2014/09/09/1593463mapa-del-dia-descubre-cuales-son-los-17-paisesmegadiversos/

2. Banco Mundial. Población total: América Latina y el Caribe[internet]. Washington D.C.: Banco Mundial;2019. [citado 12 de junio de 2020]. Disponible en: https://datos.bancomundial.org/indicator/SP.POP. TOTL

3. Organización de las Naciones Unidas para la Alimentación y la Agricultura. Oficina Regional de la FAO para América Latina y el Caribe. El hambre afecta a 42,5 millones de personas en América Latina y el Caribe. [Internet]. Roma: FAO;2020. [citado 12 de junio de 2020]. Disponible en: http://www.fao.org/americas/noticias/ver/es/c/12 $\underline{01490 /}$

4. Organización de las Naciones Unidas para la Alimentación y la Agricultura. Organización Panamericana de la Salud. Programa Mundial de Alimentos. Panorama de la seguridad alimentaria y 
nutricional en América Latina y el Caribe. [Internet]. Santiago de Chile: FAO; 2019. [citado 12 de junio de 2020]. Disponible en: https://www.onu.org.mx/wpcontent/uploads/2020/02/WFP-0000110290.pdf

5. Oficina de la Naciones Unidas Contra la Droga y el Delito. Resumen conclusiones y consecuencias en materia de políticas: informe mundial sobre las drogas [Internet]. Washington: D.C.: UNODC 2019. [citado 12 de junio de 2020]. Disponible en: https://wdr.unodc.org/wdr2019/prelaunch/WDR20 19 B1 S.pdf

6. Sen A. Desarrollo y libertad [Internet]. Barcelona: Planeta; 2000. [citado 12 de junio de 2020]. Disponible en: https://medhc16.files.wordpress.com/2018/06/des arrollo-y-libertad-amartyasen.pdf

7. García E. Bartolomé de las Casas y los derechos humanos. En: Maceiras M, Méndez L, coordinadores. Los Derechos Humanos en su origen. La República Dominicana y Antón de Montesinos. [Internet]. Salamanca: Editorial San Esteban; 2011. P. 81-114. [citado 12 de junio de 2020]. Disponible en: https://eprints.ucm.es/12666/1/bartolome de las casas.pdf

8. Galeano E. Las venas abiertas de América Latina. [Internet]. México: Siglo XXI; 1971. [citado 12 de junio de 2020]. Disponible en: https://static.telesurtv.net/filesOnRFS/news/2015/ 04/13/las venas abiertas de america latina.pdf

9. Organización de las Naciones Unidas. 1941: Declaración Palacio de St. James. [Internet].N.Y.:1941. [citado 12 de junio de 2020]. Disponible en:

https://www.un.org/es/sections/history-unitednations-charter/1941-declaration-st-jamespalace/index.html

10. Organización de las Naciones Unidas. 1941: Carta del Atlántico. [Internet]. N:Y: ONU; 1941. [citado 12 de junio de 2020]. Disponible en: https://www.un.org/es/sections/history-unitednations-charter/1941-atlantic-charter/index.html 11. Organización de las Naciones Unidas. 1945: Conferencia de San Francisco. [Internet]. N:Y:: ONU; 1945. [citado 12 de junio de 2020]. Disponible en: https://www.un.org/es/sections/history-unitednations-charter/1945-san-franciscoconference/index.html

12. Organización de Naciones Unidas-Bolivia. FAOOrganización d las Naciones Unidas para la alimentación y la agricultura [Internet]. Bolivia: ONU; 2020. [citado 12 de junio de 2020]. Disponible en: $\quad$ http://www.nu.org.bo/agencia/organizacionde-las-naciones-unidas-para-la-agricultura-y-laalimentacion/

13. Gutiérrez-Garza E. De las teorías del desarrollo al desarrollo sustentable: historia de la constitución de un enfoque multidisciplinario. Trayectorias [Internet]. 2007[citado 12 de junio de 2020];9(25):45-60. Disponible en: https://www.redalyc.org/pdf/607/60715120006.pd f

14. Prebisch R. El desarrollo económico de la América Latina y algunos de sus principales problemas. En: Cincuenta años de pensamiento en la CEPAL: textos seleccionados, vol. 1. [Internet]. México: Fondo de Cultura Económica; 1998. [citado 12 de junio de 2020]. Disponible en: file:///C:/Users/BLANCA\%20ANDREA\%200RTEGA/ Downloads/S33098N962Cvol1 es.pdf

15. Lewis WA. Desarrollo económico con oferta ilimitada de mano de obra. El trimestre económico [Internet]. 1960 [citado 12 de junio de 2020];27(4):108. Disponible en: http://132.247.149.7/moodle/pluginfileTeoría del desarrollo.php $/ 13079 / \mathrm{mod}$ resource/content/0/C AP.V.EL\%20CRECIMIENTO\%20CON\%20OFERTA\%20 ILIMITADA\%20DE\%20MANO\%20DE\%200BRE\%20D E\%20W.\%20ARTHUR\%2OLEWIS.pdf

16. Solow RM. A contribution to the Theory of economic growth. Q JEcon [Internet]. 1956[citado 5 
de mayo de 2020];70(1): 65-94. Disponible en: http://piketty.pse.ens.fr/files/Solow1956.pdf

17. Rostow W. Las etapas del crecimiento económico. México: Fondo de Cultura Económica; 1960.

18. Furtado C. Subdesarrollo y estancamiento en América Latina, Buenos Aires: Editorial Universitaria; 1966.

19. Cardoso FH, Faletto E. Dependencia y desarrollo en América Latina. [Internet]. México: Siglo XXI. 1969. Serie Documentos Teóricos No. 1. [citado 5 de mayo de 2020]. Disponible en: https://repositorio.iep.org.pe/bitstream/IEP/1027/ 1/Cardoso Faletto Dependencia-desarrolloAm\%C3\%A9rica-Latina.pdf

20. Pinto A. Notas sobre estilos de desarrollo en América Latina. Revista de la CEPAL [Internet]. 2008 [citado 23 de abril de 2020]; 96:73-93. Disponible en:

https://repositorio.cepal.org/handle/11362/11281

21.Sunkel O, Paz P. El subdesarrollo latinoamericano y la teoría del desarrollo [Internet]. México: Siglo veintiuno; 1970. [citado 23 de abril de 2020 ]. Disponible en: https://repositorio.cepal.org/bitstream/handle/113 62/1604/S33098I59S1_es.pdfEl

22. Gunder-Frank A. Gunder Frank A. Capitalismo y subdesarrollo en América Latina. [Internet] Buenos Aires: Siglo XXI; 1965[citado 23 de abril de 2020 ]. Disponible

http://www.archivochile.cl/Ideas Autores/gunderf a/gunderfa0006.pdf

23. Dos Santos, T. Dependencia y cambio socia. [Internet]. México: Plaza \& Janés; 2002. [citado 23 de abril de 2020]. Disponible en: http://theomai.unq.edu.ar/conflictos sociales/The otonio Dos Santos Teor\%C3\%ADa de la Depend encia.pdf

24. Marini RM. Dialéctica de la dependencia. [Internet]. 11a.reimp. México: Era; 1973. [citado 23 de abril de 2020 ]. Disponible en: https://n9.cl/2bvin
25. Quijano A. Imperialismo, clases sociales y Estado en el Perú: 1890-1930. Lima: Mosca Azul Editores; 1978. [citado 23 de abril 2020]. Disponible en: https://n9.cl/evhib

26. Bambirra V. Teoría de la dependencia: una anticrítica.[Internet]. México: Era. 1978 [citado 23 de abril 2020]. Disponible en: https://n9.cl/igzvq 27. Ortega-Marín BA. Las condiciones de vida de la población indígena de Xajay, Qro: su percepción del futuro y su desarrollo. [Tesis en línea]. México: Instituto Politécnico Nacional; 2015. [citado 5 de mayo de 2020 ]. Disponible en: https://n9.cl/u44gn 28. Boyer R. La teoría de la regulación: un análisis critico[Internet]. Valencia: Edicions Alfons El Magnànim; 1992. [citado 23 de abril de 2020]. Disponible en: https://dialnet.unirioja.es/servlet/libro?codigo=911 $\underline{36}$

29. Gajst N. La escuela francesa de la regulación: una revisión crítica. Visión de Futuro [Internet]. 2010 [citado 23 de abril 2020]; 1(13):31-45. Disponible en:

http://www.scielo.org.co/scielo.php?script=sci nlin ks\&ref $=000153 \&$ pid $=$ S1657-

7027201400010000300023\&lng=en

30. Comisión Económica para América Latina y el Caribe. Estudio Económico de América Latina y El Caribe 1997-1998. Chile: CEPAL; 1998. [citado 5 de mayo de 2020]. Disponible en: file:///C:/Users/BLANCA\%20ANDREA\%200RTEGA/ Downloads/EEAL1997-1998 es.pdf

31. González JE, Zapata R, Anguiano MA. Migración centroamericana en tránsito por México [Internet]. En: Consejo Nacional de Población. La situación demográfica en México. México: Consejo Nacional de Población; 2016. [citado 5 de mayo de 2020]. Disponible en: https://www.gob.mx/cms/uploads/attachment/file 232085/10 Fagoaga Zapata Anguiano.pdf

32. Naciones Unidas. Comisión Económica para América Latina y el Caribe. Perspectivas económicas 
de América Latina 2018: repensando las instituciones para el desarrollo [Internet]. Santiago: CEPAL; 2018. [citado 5 de mayo de 2020]. Disponible

https://www.cepal.org/es/publicaciones/43513-

perspectivas-economicas-america-latina-2018-

repensando-instituciones-desarrollo

33. Comisión Económica para América Latina y el Caribe. Informe Especial COVID-19. Enfrentar los efectos cada vez mayores del COVID-19 para una reactivación con igualdad: nuevas proyecciones [Internet]. Santiago: CEPAL; 2020. [citado 5 de mayo de 2020]. Informe especial COVID-19 No. 5. Disponible en: https://repositorio.cepal.org/bitstream/handle/113 62/45782/1/S2000471 es.pdf

34. Organización de las Naciones Unidas. El número de pobres en la América Latina puede crecer en 35 millones por el coronavirus COVID-19. 2020 [Internet]. Washington D.C.: ONU; 2020. [citado 5 de mayo de 2020]. Disponible en: https://news.un.org/es/story/2020/03/1471522

35. Organización de las Naciones Unidas, Departamento anuncios económicos y sociales. Ocho amenazas a la economía mundial. [Internet]. Nueva York: ONU; 2019. [citado 5 de mayo de 2020].

Disponible en:

https://www.un.org/development/desa/es/news/p olicy/world-economic-situation-and-prospectswesp-2019.html

36. Comisión Económica para América Latina y el Caribe. Panorama multidimensional del desarrollo urbano en América Latina y el Caribe. [Internet]. Santiago: CEPAL; 2017. [citado 5 de mayo de 2020 ]. Disponible en:

https://repositorio.cepal.org/bitstream/handle/113 62/41974/1/S1700257 es

37. Comisión Económica para América Latina y el Caribe. Panorama fiscal de América Latina y el Caribe 2016: las finanzas públicas ante el desafío de conciliar austeridad con crecimiento e igualdad
[Internet]. Santiago: CEPAL; 2016. [citado 5 de mayo de 2020]. Disponible en:

https://www.cepal.org/es/publicaciones/39939panorama-fiscal-america-latina-caribe-2016-

finanzas-publicas-desafio-conciliar

38. Canales Cerón Al, Rojas Wiesner ML. Panorama de la Migración Internacional en México y Centroamérica. Serie Población y Desarrollo [Internet]. 2018[citado 5 de mayo de 2020];(124). Disponible en: https://repositorio.cepal.org/bitstream/handle/113 62/43697/1/S1800554 es.pdf

39. Nieto Parra S. Más allá del PIB, para un desarrollo sostenible e inclusivo en América Latina [Internet]. Santo Domingo: CEPAL; 2018[citado 5 de mayo de 2020]. Disponible en: http://economia.gob.do/wpcontent/uploads/drive/Publicaciones/OCDE\%203\% 20de\%20abril\%202018\%20MEPD.pdf

40. Comisión Económica para América Latina y el Caribe. Panorama social de América Latina [Internet]. Santiago: CEPAL; 2019. [citado 5 de mayo de 2020]. Disponible en: https://repositorio.cepal.org/bitstream/handle/113 62/44969/5/S1901133_es.pdf

41. Banco Mundial. Latinoamérica Indígena en el Siglo XXI [Internet]. Washington: BM; 2015. [citado 5 de mayo de 2020]. Disponible en: http://documents1.worldbank.org/curated/en/541 651467999959129/pdf/Latinoam\%c3\%a9rica-

ind\%c3\%adgena-en-el-siglo-XXI-primera-

d\%c3\%a9cada.pdf

42. Comisión Económica para América Latina y el Caribe. La inversión extranjera directa en América Latina y el Caribe [Internet]. Santiago: CEPAL; 2019 [citado 30 de mayo de 2020]. Disponible en: https://www.cepal.org/es/publicaciones/44697-lainversion-extranjera-directa-america-latina-caribe2019

43. Comisión Económica para América Latina y el Caribe. Panorama fiscal de América Latina y el 
Caribe [Internet]. Santiago: CEPAL; 2018 [citado 30 de mayo de 2020]. Disponible en: https://www.cepal.org/es/publicaciones/43405panorama-fiscal-america-latina-caribe-2018desafios-politicas-publicas-marco-la

44. Marrero A. La sociedad del conocimiento: una revisión teórica de un modelo de desarrollo para América Latina . Arxius de ciències socials [Internet]. 2007[citado 30 de mayo de 2020];(17):63-73. Disponible

en:

https://roderic.uv.es/handle/10550/19447

45. Palma A. Malnutrición en niños y niñas en América Latina y el Caribe. Bol Desafíos [Internet]. 2018[citado 30 de mayo de 2020];21. Disponible en: https://www.cepal.org/es/enfoques/malnutricionninos-ninas-america-latina-caribe

46. Vidal de la Rosa G. De Cive. Una revisión de la agenda de la participación política en América Latina. Rev Debates [Internet]. 2016 [citado 23 de abril de 2020];10:(1):71-88. Disponible en: https://pdfs.semanticscholar.org/2103/6bc79d64e Odb6ed6a83bb1ca2d9e3a708dca.pdf

47. Hernández Suarez, JL. Política Migratoria de México hacia Centroamérica y presión Estadounidense. CLIVATGE [Internet]. 2019[citado 30 de mayo de 2020];(7):8-45. Disponible en: https://revistes.ub.edu/index.php/clivatge/article/v iewFile/28595/29186

48. Maddaloni D. Para una sociología de la violencia. América latina en perspectiva comparada. Cultura Latinoamericana. Cultura Latinoam. [Internet]. 2016[citado 30 de mayo de 2020];24(2):110-128. Disponible

https://editorial.ucatolica.edu.co/index.php/RevCla t/article/view/1592/1470

49. Organización Panamericana de la Salud. Políticas públicas y marcos legales para la prevención de la violencia relacionada con adolescentes y jóvenes Políticas públicas y marcos legales para la prevención de la violencia relacionada con adolescentes y jóvenes [Internet]. Washington: OPS;
2006. [citado 30 de mayo de 2020]. Disponible en: https://www.paho.org/spanish/ad/fch/ca/ca politi cas.violencia.pdf

50. di Napoli PN. Una mirada a las investigaciones cualitativas sobre jóvenes, conflictos y violencia en las escuelas secundarias de América Latina. Entre Diversidades. Rev Cienc soc y humanidades [Internet]. 2018 [citado 30 de mayo de 2020];(10):937.

Disponible

en:

https://www.redalyc.org/articulo.oa?id=45595967 4001

51. Rodgers D, Baird A. Entender a las pandillas de América Latina: una revisión de la literatura. Estud Socio Juríd [Internet]. 2016[citado 30 de mayo de 2020];18(1):13-53. Disponible en: https://www.redalyc.org/pdf/733/73343370001.pd f

52. Zaffaroni RE. Violencia letal en América Latina. Cuad Derecho Penal [Internet]. 2015 citado 30 de mayo de 2020];(13):57-76. Disponible en: https://revistas.usergioarboleda.edu.co/index.php/ cuadernos de derecho penal/article/view/455/52 $\underline{3}$

53. Moloeznik MP, Trefler P. América Latina: violencia en México y Centroamérica. Anuario Latinoam Cienc Pol Relac Internacionales [Internet]. 2017[citado 30 de mayo de 2020];4:13-19. Disponible en: https://journals.umcs.pl/al/article/view/5409 54. Monsálvez Araneda DG. Perspectivas sobre la violencia política en la historia reciente de américa latina: aspectos historiográficos, teóricos, metodológicos y estudios de casos. Folia Hist Nordeste [Internet]. 2018[citado 30 de mayo de 2020];(32):79-81. Disponible en: https://revistas.unne.edu.ar/index.php/fhn/article/ view/3497

55. Programa de las Naciones Unidas para el Desarrollo Informe regional de desarrollo humano 2013-2014 seguridad ciudadana con rostro humano: diagnóstico y propuestas para América 
Latina [Internet]. New York: PNUD; 2013 [citado 23 de abril de 2020]. Disponible en: https://www.latinamerica.undp.org/content/rblac/ es/home/library/human development/informeregionalde-desarrollo-humano2013-2014-.html

56. Programa de las Naciones Unidas para el Desarrollo. Informe sobre desarrollo humano 2019 más allá del ingreso, más allá de los promedios, más allá del presente: desigualdades del desarrollo humano en el siglo XXI [Internet]. New York: PNUD; 2019. [citado 23 de abril de 2020]. Disponible en:http://hdr.undp.org/sites/default/files/hdr 201 9 overview - spanish.pdf

57. Organización Internacional del Trabajo. OIT: cerca de 140 millones de trabajadores en la informalidad en América Latina y el Caribe [Internet]. Ginebra: OIT; 2018. [citado 23 de abril de 2020].

Disponible

en:

https://www.ilo.org/americas/sala-de

prensa/WCMS 645596/lang--es/index.htm.

58. Martínez Alier J. Atlas de Justicia Ambiental [Internet]. Barcelona: ICTA-UAB; 2014. [citado 30 de mayo de 2020]. Disponible en: https://ejatlas.org/ 59. Organización de las Naciones Unidas para la Alimentación y la Agricultura. El estado de la seguridad alimentaria y la nutrición en el mundo: protegerse frente a la desaceleración y el debilitamiento de la economía [Internet]. Roma: FAO; 2019[citado 30 de mayo de 2020]. Disponible en: http://www.fao.org/3/ca5162es/ca5162es.pdf 60. Ferreyra MM, Avitabile C, Botero Álvarez J, Haimovich Paz F, Urzúa S. Momento decisivo: la educación superior en América Latina y el Caribe [Internet]. Washington: BM; 2017. [citado 30 de mayo de 2020]. Disponible en: https://openknowledge.worldbank.org/bitstream/h andle/10986/26489/211014ovSP.pdf?sequence $=5$ \&isAllowed $=y$

61. Gómez Sabatini JC, Jiménez JP, Martner R. Consensos y conflictos en la política tributaria de América Latina [Internet]. Santiago: CEPAL; 2017. [citado 30 de mayo de 2020]. Disponible en: https://repositorio.cepal.org/bitstream/handle/113 62/41048/6/S1700003 es.pdf

62. Cecchini S. Protección social universal en América Latina y el Caribe: textos seleccionados 2006-2019 [Internet]. Santiado: CEPAL; 2019. [citado 30 de mayo de 2020]. Disponible en: https://www.cepal.org/es/publicaciones/44995proteccion-social-universal-america-latina-caribetextos-seleccionados-2006-2019

63. Comisión Económica para América Latina y el Caribe. Acuerdo regional sobre el acceso a la información, la participación pública y el acceso a la justicia en asuntos ambientales en América Latina y el Caribe [Internet]. Santiago: CEPAL; 2018. [citado 30 de mayo de 2020]. Disponible en: https://repositorio.cepal.org/bitstream/handle/113 62/43595/1/S1800429 es.pdf

64. Gamiño Muñoz R. Memorias de la violencia política en América Latina: tensiones y complementariedades. Hist Graf [Internet]. 2019. [citado 30 de mayo de 2020]. [consultado];26(52):267-299. Disponible en: http://www.scielo.org.mx/pdf/hg/n52/1405-0927hg-52-267.pdf

65. Kruijt D. Violencia y pobreza en América Latina: los actores armados. Pensam Iberoam [Internet]. 2008[citado 30 de mayo de 2020]; 2:55-70. Disponible en: https://dialnet.unirioja.es/servlet/articulo?codigo= $\underline{2873318}$

66. García K. Poverty, gender and violence in the narratives of former narcos: accounting for drug trafficking violence in Mexico [Internet]. England: University of Bristol; 2018. [citado 30 de mayo de 2020]. Disponible en: https://researchinformation.bris.ac.uk/en/studentTheses/povertygender-and-violence-in-the-narratives-of-former-

$\underline{\text { narcos }}$

67. Chioda L. Fin a la violencia en América Latina: una mirada a la prevención desde la infancia a la 
edad adulta [Internet]. Washington: BM; 2016. [citado 30 de mayo de 2020]. Disponible en: https://openknowledge.worldbank.org/bitstream/h andle/10986/25920/210664ovSP.pdf

68. Beltrán Savenije MA. Criminología feminista. Estado del arte y presencia en Latinoamérica [Internet]. La Plata: UNLP; 2010. [citado 30 de mayo de 2020]. Disponible en: http://www.memoria.fahce.unlp.edu.ar/trab even tos/ev.5515/ev.5515.pdf

69. Herrera Cortés MC, Olaya Gualteros V, Urrego Salas AF. Configuración de subjetividades y violencia política en América Latina. Aportes a la emergencia y consolidación de un campo de estudios. Estudios Políticos [Internet]. 2019[citado 30 de mayo de 2020];(56):249-268. Disponible en: https://revistas.udea.edu.co/index.php/estudiospol iticos/article/view/336046

70. López V, Ascorra P, Litichever L, Ochoa A. Violencia y convivencia escolar en América Latina: políticas, prácticas, marcos de comprensión y acción. Psicoperspectivas [Internet]. 2019 [citado 30 de mayo de 2020];18(1):1-8. Disponible en: https://www.psicoperspectivas.cl/index.php/psicop erspectivas/article/viewFile/1608/963

71. Jiménez Rodrigo ML, Guzmán Ordaz R. El caleidoscopio de la violencia contra las mujeres en la pareja desde la desigualdad de género: una revisión de enfoques analíticos. Rev Estud Soc [Internet]. 2015[citado 30 de mayo de 2020];(54):93-106. Disponible en: http://journals.openedition.org/revestudsoc/9488 72. Briceño-León R. La nueva violencia urbana de América Latina [Internet]. En Violencia, sociedad y justicia en América Latina. Buenos Aires: CLACSO; 2002. 13-26. [citado 30 de mayo de 2020]. Disponible en: http://biblioteca.clacso.edu.ar/gsdl/collect/clacso/i ndex/assoc/D3078.dir/1intro.pdf 